\title{
Anticoccidial Effect of CS 32 Compounds Against Eimeria tenella Infection in Chickens
}

\author{
Kwangsik Jang ${ }^{1,2 \#}$, Se Eun Kim ${ }^{1,2 \#}$, Kyung Mi Shim ${ }^{1,2}$, Hye Ji Park ${ }^{1,2}$, Suk Kim ${ }^{3}$, Wongi Min ${ }^{3}$, Jin Cheol Yoo ${ }^{4}$ \\ and Seong Soo Kang ${ }^{1,2 *}$
}

1Department of Veterinary Surgery, College of Veterinary Medicine, BK21 Plus Project Team, Chonnam National University, Gwangju 61186, Korea

${ }^{2}$ Biomaterial R\&BD Center, Chonnam National University, Gwangju 61186, Korea

${ }^{3}$ Institute of Animal Medicine, College of Veterinary Medicine, Gyeongsang National University, Jinju 52828, Korea

${ }^{4}$ Department of Pharmacy, College of Pharmacy, Chosun University, Gwangju 61452, Korea

\#These authors contributed equals to this study.

Corresponding

Seong Soo Kang, DVM, PhD

College of Veterinary Medicine, Chonnam

National University, 77, Yongbong-ro,

Buk-gu, Gwangju 61186, Korea

Phone : +82-62-530-2877

Fax : +82-62-530-2881

E-mail : vetkang@chonnam.ac.kr .

Received : February 19, 2020

Revised : March 09, 2020

Accepted : March 11, 2020

No potential conflict of interest relevant to this article was reported.

Copyright (C) 2020 Journal of Bacteriology and Virology

(C)This is an Open Access article distributed under the terms of the Creative Commons Attribution Non-Commercial License (http://creativecommons.org/ license/by-nc/3.0/).
Coccidiosis-causing Eimeria species are transmitted in poultry via the oral-fecal route and can lead to hemorrhagic diarrhea and mortality. This results in enormous economic losses in the poultry industry. Furthermore, its resistance to some currently used antibiotics is increasing. This has prompted the development of new alternative drug therapies that address the issue of chemical-free meat production. Effective management of infectious diseases in veterinary practice includes the induction of protective and adaptive immunity by treatment with an alternative agent. In this study, we evaluated the anticoccidial effects of dietary supplementation of Chosun University (CS) 32 compounds $(0.1 \%$ and $1.0 \%$ ) against Eimeria tenella, which was isolated and purified from the supernatant of culture broth of Bacillus strain (KCTC18250P), as well as its effect on the growth rate and feed efficiency in chickens. Overall, we observed a decrease in lesion scores and oocyte output in CS 32 compounds-treated chickens. We concluded that $0.1 \%$ CS 32 compounds displayed anticoccidial effects against $E$. tenella infection.

Key Words: CS 32 compounds, anticoccidial effect, E. tenella, chickens

\section{INTRODUCTION}

Coccidiosis in chickens is a serious intestinal disease caused by 7 different species of Eimera protozoan parasites, which leads to significant losses in the poultry industry (1). Its mode of transmission is widely reported as an oro-fecal route. A total of 8 sporozoites are released from the 4 sporocysts contained within each oocyst upon ingestion. These rapidly adhere to the intestinal epithelium of the host. The invasion begins with a limited number of asexual cycles and the merozoites rapidly amplify. Eventually, after the merozoites are sexually differentiated, the male gametes fertilize the female macrogametes to create oocytes that are excreted in feces (2). E. tenella infection can lead to the development of hemorrhagic diarrhea, subsequent weight loss, and often death (2). As an obligate intracellular protozoan parasite, E. tenella possess a complex life cycle that is completed within 7 days. During this period, it undergoes intracellular development permitting distinctive 
intracellular stages to proliferate within the cecal epithelium (3). Drug residues in poultry products have become an increasing concern with the emergence of multiple drugs for coccidiosis control. Concomitantly, the development of drug resistance has created a growing pressure within the government and consumer groups to evaluate, regulate, and even ban these drugs (2). Vaccination has been used in coccidiosis control for a long time; however, its application has been limited due to factors related to cost, safety, and the need for farmers or veterinarians to have technical expertise in administering vaccines (4). Thus, developing alternative drug-independent control strategies for avian coccidiosis is imperative.

Chosun University (CS) 32 compounds are food supplements, which are isolated from the culture medium of a new strain of Bacillus, Bacillus spp. strain CS 32, with a molecular weight of 5697.6 Da as determined by tricine-sodium dodecyl sulfate-polyacrylamide gel electrophoresis (tricine SDS-PAGE) (5). Previous studies demonstrated the probiotic effect of CS 32 compounds as antibiotic substitutes (6). Probiotic-containing diets can modulate immunity and are used as alternatives to antibiotics in Korean native chickens (7). However, the effects of CS 32 compounds as an alternative supplement against Eimeria infection have not been evaluated in vivo. In this study, we investigated the anticoccidial effect of CS 32 compounds in chickens infected with E. tenella.

\section{MATERIALS AND METHODS}

\section{Isolation and production of biologically active antimicrobial peptides from Bacillus spp. CS 32}

\section{A. Optimization of the fermentation process}

Bacillus strain CS 32 (KCTC18250P), which was isolated from the traditional Korean food (Kimchi), was used in this experiment to isolate biologically active antimicrobial peptide $(5,6)$. The antimicrobial peptide production of the strain was optimized by several culture parameters, such as the carbon level, nitrogen source, and mineral content. The effect of various carbon sources on antimicrobial peptide production was determined by combining $1 \%$ yeast extract with supplements such as glucose, mannitol, starch, lactose, fructose, sorbitol, sucrose, and maltose (1\% each) in the media. Fermentation was performed in $250 \mathrm{~mL}$ Erlenmeyer flasks containing $50 \mathrm{~mL}$ of media with steady shaking at $180 \mathrm{rpm}$. Subsequently, the influence of nitrogen sources on peptide production was determined by using medium containing $1 \%$ glucose as the carbon source, combined with supplements such as beef extract, malt extract, tryptone, yeast extract, oatmeal, soytone, and peptone (1\% each). In addition, the percentage of carbon and nitrogen sources to peptide production was valued as an optimized medium, including the best carbon sources ( $1 \%$ or $2 \%$ glucose), and the best nitrogen sources $(0.5 \%$ or $1 \%$ beef extract and peptone) (6).

\section{B. Preparation of Bacillus spp. CS 32-producing antimicrobial peptides}

Bacillus spp. CS 32 was cultured in an optimized medium ( $1 \%$ glucose, $0.5 \%$ beef extract, and $0.5 \%$ peptone) at $37^{\circ} \mathrm{C}$ and $180 \mathrm{rpm}$ for $60 \mathrm{~h}$. The cells were separated by centrifugation $\left(6000 \times \mathrm{g} \mathrm{rpm}, 30 \mathrm{~min}, 4^{\circ} \mathrm{C}\right)$, and peptides were precipitated from the supernatant at $4^{\circ} \mathrm{C}$ overnight with ammonium sulfate of $60 \%$ saturation. The precipitate was collected by centrifugation $\left(6000 \times \mathrm{g} \mathrm{rpm}, 60 \mathrm{~min}, 4^{\circ} \mathrm{C}\right)$, resuspended in $10 \mathrm{mM}$ Tris- $\mathrm{HCl}$ buffer $(\mathrm{pH} 8.0)$ and dialyzed using a 1-kDa cutoff membrane (Merk Millipore, Burlinton, MA, USA). The crude extracts were applied to a Sepharose CL-6B column $(2.2 \times 116 \mathrm{~cm})$ (GE Healthcare, Chicago, IL, USA) and next to a Sephadex G-50 column $(1.5 \times 70 \mathrm{~cm})$ (GE Healthcare, Chicago, IL, USA), and eluted with the same buffer. Active fractions were pooled and concentrated using a YM1 amicon filter (Merck KGaA, Darmstadt, Germany) and stored at $-20^{\circ} \mathrm{C}(6)$

The CS 32 compounds used as anticoccidial food were supplied in the standard diet for 10 days before infection with $E$. tenella. The chickens were given a supplemental diet until the experiment was over. Starter diets using corn and soybeans, 
which are commonly used in Korea and include corn as the major cereal and soybean meals made of protein concentrate, meet the National Research Council nutritional requirements for broiler chickens (NRC, 1994). The formulated starter diets consisted of $53.44 \%$ corn, $33.65 \%$ soybean meal, $4.68 \%$ soybean oils, $4.16 \%$ corn gluten meal, and $3.57 \%$ other ingredients. The calculated analysis value of the diets was $3100 \mathrm{kcal} / \mathrm{kg}$ metabolizable energy, $22 \%$ crude protein, $1.10 \%$ lysine, $1.00 \%$ calcium, $0.87 \%$ methionine plus cysteine, $0.50 \%$ available protein, and $0.05 \%$ methionine

\section{Animal and animal experiments}

Male Korean native chickens and female Rhode Island Red chickens were mated and fertilized eggs were obtained. The chickens were hatched from those eggs at the National Livestock Research Institute (Daejeon, Korea). Each group of chickens was bred with a commercial anticoccidial agent-free broiler feed and had access to coccidian-free water. Continuous 24-hour artificial lighting was maintained during the experiment. The initial temperature on the first day was set at $31 \sim 32^{\circ} \mathrm{C}$ and lowered by $2^{\circ} \mathrm{C}$ per week until the experiment was completed. All the animal experiments conducted in this study were reviewed and approved by the Animal Ethical Committee of Gyeongsang National University (Authorization Number GNU120615-(0022).

The 112-day-old chicks were divided into four groups of 28 chickens each, and then randomly divided into four subgroups; the subgroups were further subdivided into four additional subgroups. In total, 16 cages containing 7 chickens each were used. Each group was treated as follows: control, uninfected and untreated $(n=28)$; untreated, infected and untreated $(n=$ 28); $0.1 \%$ CS 32 compounds, infected and treated with $0.1 \%$ CS 32 compounds ( $n=28) ; 1.0 \%$ CS 32 compounds, infected and treated with $1.0 \%$ CS 32 compounds $(n=28)$.

The chickens did not receive any vaccinations during the experiment. All treatments were initiated 10 days before the infection and sustained throughout the experiment.

The chickens were infected with E. tenella (Korean isolate 291-7) (8) and E. tenella oocysts were spread into specific pathogen-free chickens. Before infection, the virulence of the incipient stock of oocysts was assessed. The feces of specific pathogen-free chickens were collected to purify the new oocytes. The oocysts were then cultivated in a $2 \%$ potassium dichromate solution in a water bath $\left(30^{\circ} \mathrm{C}\right)$ for $48 \mathrm{~h}$ to induce sporulation. After sporulation, the oocysts were maintained at $4^{\circ} \mathrm{C}$ for 4 weeks. Just before infection, the oocysts were cleaned by dipping them into a $5.25 \%$ sodium hypochlorite solution. Then, the oocysts were washed in sterile phosphate-buffered saline three times. The counted oocysts were diluted to $1 \times 10^{5}$ oocysts $/ \mathrm{mL}$, the final concentration of the sporulation. A $5 \mathrm{~cm}$ esophageal cannula with $5 \times 10^{4}$ sporulated $E$. tenella oocysts was inserted into the crop of ten-day-old chicks for oral infection. The number of oocysts per gram of feces (OPG) was calculated by the following formula:

OPG = oocyst count from days 6 to 10 p.i. $\times$ dilution factor $\times($ fecal sample volume / counting chamber volume) / number of birds per cage

\section{Evaluation of drug treatment efficacy}

We evaluated the efficacy of CS 32 compounds as an anticoccidial food supplement using the following indicators: body weight gain (BWG) and lesion scores (9).

The chickens were weighed every 5 days during the experiment to analyze BWG, i.e. at day 10 pre-infection and at days 0,5 , and 10 post infection (p.i.).

Seven chickens from each group were randomly selected and sacrificed on day 5 p.i. to determine the lesion scores. The cecum of each chicken was evaluated and the severity of the lesions was scored as 1 of 5 ranks (between 0 and 4 ) based on 
the epithelial color, fluid accumulation, and overall general appearance of the intestine (serosal thickness, mucosal erosion, dilation, and similar factors) according to the method described by Johnson \& Reid (Table 1) (10).

A total of 112 chickens in 4 subgroups (28 chickens per group) were measured to obtain the BWG data (day 5 p.i.). After sacrificing 7 chickens from each group on day 5 p.i., the remaining 84 chickens were analyzed to obtain the OPG data for the 4 subgroups, each by day 10 p.i.

Table 1. Chicken lesion score technique for $E$. tenella infection by Johnson and Reid

\begin{tabular}{cc}
\hline Score & Description \\
\hline 0 & No gross lesions \\
+1 & Very few scattered petechiae on the cecal wall; no thickening of the cecal walls; \\
normal cecal contents present
\end{tabular}

\section{Statistical analysis}

A general linear model procedure of SAS software (SAS Institute, Inc., Cary, NC, USA) was used to statistically analyze the mean and analysis of variance for BWG, lesion scores, and oocyst excretion. After natural log transformation, we analyzed the group OPGs to modify the heterogeneity of variance and to obtain, approximately, a normal distribution data. For estimating the significance of the differences between the 4 groups, a Mood-Brown median test was used to analyze lesion scores and oocyst excretion.

Mean values \pm standard deviation for the different groups were used as results of measured indicators at different time periods throughout the experiment. Using GraphPad Prism ${ }^{\mathrm{TM}}$ software (GraphPad Software, San Diego, CA, USA), differences were considered significant at $P<0.05$.

\section{RESULTS}

\section{Evaluation of BWG}

We examined the effect of CS 32 compounds on E. tenella inhibition by BWG. There were no significant differences in BWG between the 4 groups from day 5 to day 10 p.i. (Fig. 1).

\section{Evaluation of oocyst output}

Fig. 2 shows the oocyst output evaluation of chickens infected with $E$. tenella from days 6 to 10 p.i. Of note, the value of OPG in the $0.1 \%$ CS 32 compounds group decreased by approximately 33\% compared to that of the untreated group (Fig. 2). However, the value of OPG in the $1 \%$ CS 32 compounds group was higher than that of either the $0.1 \%$ CS 32 compounds group or the untreated group (Fig. 2). The patterns of oocyst output in the $0.1 \%$ CS 32 compounds and $1.0 \%$ CS 32 compounds groups differed from those in the untreated groups. 


\section{Evaluation of lesion scores}

After infection, lesion scores in the $0.1 \%$ CS 32 compounds and $1.0 \%$ CS 32 compounds groups were higher than those in the uninfected group; however, the $0.1 \%$ CS 32 compounds group was significantly lower than the untreated group when compared to the increasing pattern seen in the $1 \%$ CS 32 compounds group (Fig. 3). Similar to the results of oocyst output, significant differences were found in lesion scores between $0.1 \%$ CS 32 compounds and $1.0 \%$ CS 32 compounds groups before infection (Fig. 3).

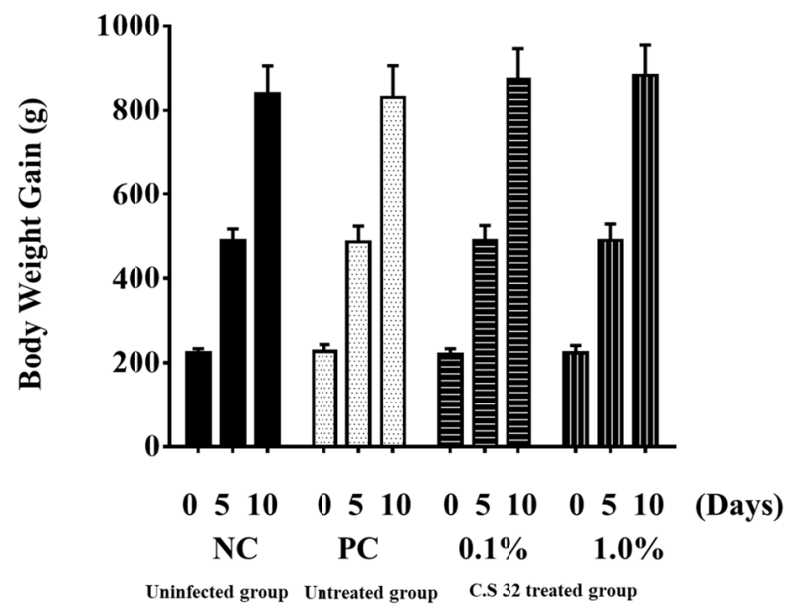

Fig. 1. Effects of dietary supplementation with CS 32 compounds on weight gains after challenged with $E$. tenella. NC: neg ative control (uninfected and untreated group); PC: positive control (infected and untreated group); $0.1 \%: 0.1 \%$ CS 32 co mpounds group (infected and treated with $0.1 \%$ CS 32 compounds); $1.0 \%: 1.0 \%$ CS 32 compounds group (infected and treated with $1.0 \%$ CS 32 compounds); $n=28$ for days 0 and 5 , and $n=21$ for days 10 .

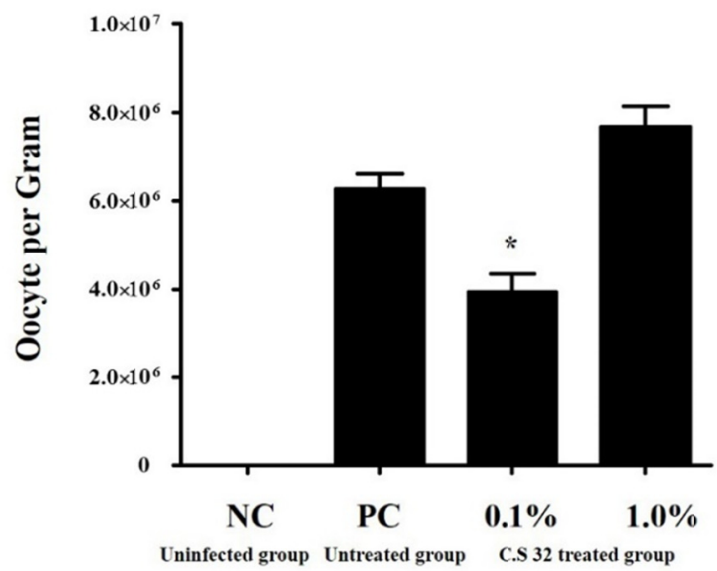

Fig. 2. Effects of dietary supplementation with CS 32 compounds on oocyte output after challenged with $E$. tenella from days 6 to 10 p.i.. NC: negative control (uninfected and untreated group); PC: positive control (infected and untreated group); $0.1 \%: 0.1 \%$ CS 32 compounds group (infected and treated with $0.1 \%$ CS 32 compounds); $1.0 \%: 1.0 \%$ CS 32 compounds group (infected and treated with $1.0 \%$ CS 32 compounds); $n=21$ for each group.

*Significant difference $(P<0.05)$ between PC and $0.1 \%$ CS 32 compounds groups. 


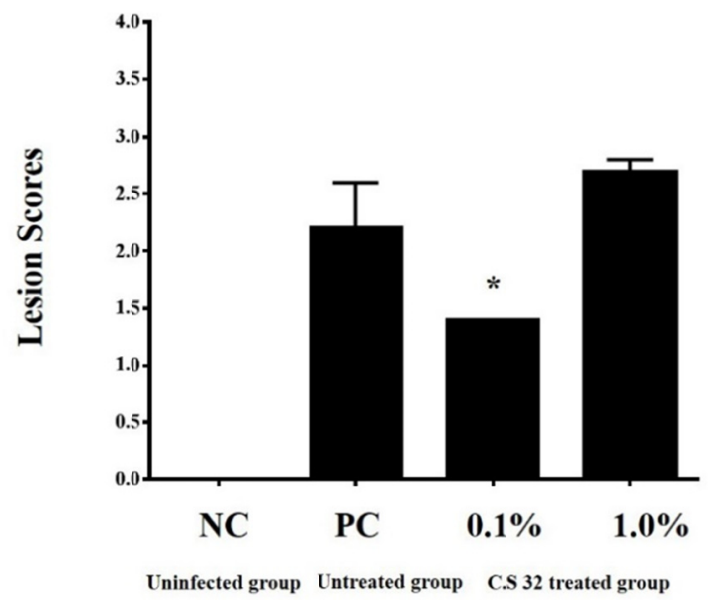

Fig. 3. Effects of dietary supplementation with CS 32 compounds on lesion score after challenged with $E$. tenella at 5 days p.i.. NC: negative control (uninfected and untreated group); PC: positive control (infected and untreatted group); $0.1 \%: 0.1 \%$ CS 32 compounds group (infected and treated with 0.1\% CS 32 compounds); $1.0 \%: 1.0 \%$ CS 32 compounds group (infected and treated with $1.0 \%$ CS 32 compounds); $n=7$ for each group.

*Significant difference $(P<0.05)$ between PC and $0.1 \%$ CS 32 compounds groups.

\section{DISCUSSION}

Recently, probiotics, prebiotics, and synbiotics have been considered the most common alternatives to antibiotics (11). With growing concerns about antibiotic-resistant bacteria, there are a wider range of restrictions on the use of veterinary antibiotic growth promoters (AGPs). Consequently, interest in prebiotic oligosaccharides and probiotic microorganisms is increasing (12). Recently, several studies have shown that probiotics improve the growth rate of chickens, and many of these probiotics had growth-promoting effects similar to those of AGPs (13). Probiotics are a living microbial food additive that cause a positive change in the intestinal microbial balance (14-17). They can improve animal food efficiency and growth performance as supplemental probiotics (18). The CS 32 compounds used in this study were produced by Bacilllus spp.; aerobic microflora such as Lactobacillus spp. have been widely used in the livestock industry (19).

Bacillus spp. have several characteristic features that make them suitable as possible biological control agents, such as availability in the soil and production of biologically-active metabolites (20) and peptide antibiotic compounds like circulin, colistin, and polymyxin (21). Bacitracin, the most important antibiotic product of Bacillus spp., mainly inhibits gram-positive bacteria (22). The aminopolyol antibiotic, zwittermicin A, may represent a new class of Bacillus antibiotics. Bacillus spp. also produce antibacterial and antifungal peptides $(23,24)$, lipopeptides $(25,26)$, and aminoglycosides $(27,28)$.

We studied the effect of Bacillus spp.-derived CS 32 compounds as a food supplement with probiotic activities for coccidiosis in chickens, because its antibacterial activity has been shown in previous studies (5). CS 32 comprounds were prepared from the culture medium of Bacillus spp. CS 32, which showed effectiveness on gram-negative and gram-positive bacteria and MRSA (6). The anticoccidial effect of CS 32 compounds was evaluated by fecal oocyst shedding and cecal lesion score.

Further experiments are needed to define effects of dietary supplementation with CS 32 compounds on weight gains, as there were no significant differences between the negative control (uninfected and untreated group) and the positive control (infected and untreated group).

Supplementation with $0.1 \%$ CS 32 compounds significantly reduced fecal oocyst output and resulted in decreased cecal lesions in chickens infected with $E$. tenella. 
Evaluating the pathogenic effects of $E$. tenella infection requires appropriate quantification of oocyst inoculation. The number of oocysts used for infection should induce statistically significant differences in the CS 32 compounds-untreated chickens and the CS 32 compounds-treated chickens. Excretion of oocysts in the feces is a parameter that is evaluated in coccidiosis. However, several researchers have disagreed on its significance and claim that counting oocysts is an unreliable and unsatisfactory way to assess the effect of anticoccidial therapy (29). Considering the reduction in fecal output of oocysts in the treated groups, CS 32 compounds treatment might correlate with an anticoccidial effect. Infection of $E$. tenella causes damage and swelling of the cecal wall and it leads to alterations in the normal function of the intestinal mucosa (30). When comparing the $0.1 \%$ CS 32 compounds-treated group with the $1.0 \%$ CS 32 compounds-treated group, OPG and lesion scores were increased in the $1.0 \%$ CS 32 compounds-treated group. This results are in accordance with those of Levine et al. (31). Treatment with $0.1 \%$ CS 32 compounds effectively lowered the lesion score caused by $E$. tenella. This finding shows that a diet supplemented with $0.1 \%$ CS 32 compounds reduces the gross pathological change induced by $E$. tenella.

Therefore, using $0.1 \%$ CS 32 compounds as a dietary supplement can improve host defense responses and have beneficial effects. Our results verified that $0.1 \%$ CS 32 compounds modulates host resistance to parasitic infections. This is the first study describing the anticoccidial effect of CS 32 compounds. However, the definite basic mechanism of CS 32 compoundsmediated immune response against coccidiosis is unclear. As concluded by lesion scores and fecal oocyst excretion, $0.1 \%$ CS 32 compounds as food supplements could effectively control $E$. tenella infection. Thus, in poultry, a CS 32 compounds-based diet may help prevent or treat coccidial infection. Further studies on other species of Coccidia are needed.

\section{ACKNOWLEDGMENTS}

This work was supported by a grant from the Next-Generation BioGreen 21 Program (No: PJ01135201), Rural Development Administration, Republic of Korea.

\section{REFERENCES}

1) McDonald V, Shirley MW. Past and future: vaccination against Eimeria. Parasitology 2009;136:1477-89.

2) Sharman PA, Smith NC, Wallach MG, Katrib M. Chasing the golden egg: vaccination against poultry coccidiosis. Parasite Immunol 2010;32:590-8.

3) Del Cacho E, Gallego M, Lillehoj HS, Quílez J, Lillehoj EP, Ramo A, et al. IL-17A regulates Eimeria tenella schizont maturation and migration in avian coccidiosis. Vet Res 2014;45:25.

4) Peek HW, Landman WJ. Coccidiosis in poultry: anticoccidial products, vaccines and other prevention strategies. Vet Q 2011:31:143-61.

5) Choi YH, Cho SS, Simkhada JR, Rahman MS, Choi YS, Kim CS, et al. A novel multifunctional peptide oligomer of bacitracin with possible bioindustrial and therapeutic applications from a Korean food-source Bacillus strain. PloS ONE 2017:12:e0176971.

6) Kim SE, Choi GH, Shim KM, Kim JC, Yo JC, Cho SS, et al. Effects of probiotic CS-32 as a feed additive on growth performance of broiler chickens. J Biomed Res 2013;14:170-6.

7) Lee SB, Kim BK, Park CH, Park GH, Jin YC, Kang HS, et al. Effects of dietary pro-biotics and immunomodulator as an alternative to antibiotics in Korean Native Chicken. J Anim Sci Technol 2011:53:409-18. 
8) Jeong J, Kim WH, Yoo J, Lee C, Kim S, Cho JH, et al. Identification and comparative expression analysis of interleukin 2/15 receptor beta chain in chickens infected with $E$. tenella. PLoS One 2012;7:e37704

9) Chapman HD, Sandstrom J, Breeding SW. Effect of the anticoccidial agents halofuginone and monensin when given with growth promoting antibiotics upon the control of coccidiosis in the turkey. Avian Pathol 1998;27:498-504.

10) Johnson J, Reid WM. Anticoccidial drugs: lesion scoring techniques in battery and floor-pen experiments with chickens. Exp Parasitol 1970;28:30-6.

11) Chu GM, Lee SJ, Jeong HS, Lee SS. Efficacy of probiotics from anaerobic microflora with prebiotics on growth performance and noxious gas emission in growing pigs. Anim Sci J 2011:82:282-90

12) Mookiah S, Sieo CC, Ramasamy K, Abdullah N, Ho YW. Effects of dietary prebiotics, probiotic and synbiotics on performance, caecal bacterial populations and caecal fermentation concentrations of broiler chickens. J Sci Food Agric 2014;94:341-8.

13) Mountzouris KC, Tsitrsikos P, Palamidi I, Arvaniti A, Mohnl M, Schatzmayr G, et al. Effects of probiotic inclusion levels in broiler nutrition on growth performance, nutrient digestibility, plasma immunoglobulins, and cecal microflora composition. Poult Sci 2010;89:58-67.

14) Fuller R. Probiotics in man and animals. J Appl Bacteriol 1989;66:365-78.

15) Kelly D. Probiotics in young and newborn animals. J Anim Feed Sci 1998;7:15-23.

16) Ko SY, Bae IH, Yee ST, Lee SS, Uuganbayar D, Oh Jl, et al. Comparison of the effect of green tea by-product and green tea probiotics on the growth performance, meat quality, and immune response of finishing pigs. Asian-Aust J Anim Sci 2008;21:1486-94

17) Ko SY, Yang CJ. Effect of green tea probiotics on the growth performance, meat quality and immune response in finishing pigs. Asian-Aust J Anim Sci 2008;21:1339-47.

18) Mohan B, Kadirvel R, Natarajan A, Bhaskaran M. Effect of probiotic supplementation on growth, nitrogen utilisation and serum cholesterol in broilers. Br Poult Sci 1996;37:395-401.

19) Taylor CC, Ranjit NJ, Mills JA, Neylon JM, Kung L Jr. The effect of treating whole-plant barley with Lactobacillus buchneri 40788 on silage fermentation, aerobic stability, and nutritive value for dairy cows. J Dairy Sci 2002;85:1793-800.

20) Silo-Suh LA, Lethbridge BJ, Raffel SJ, He H, Clardy J, Handelsman J. Biological activities of two fungistatic antibiotics produced by Bacillus cereus UW85. Appl Environ Microbiol 1994;60:2023-30.

21) Katz E, Demain AL. The peptide antibiotics of Bacillus: chemistry, biogenesis, and possible functions. Bacteriol Rev 1977:41:449-74

22) Amin A, Khan MA, Ehsanullah M, Haroon U, Azam SM, Hameed A. Production of peptide antibiotics by Bacillus sp. GU 057 indigenously isolated from saline soil. Braz J Microbiol 2012;43:1340-6.

23) Sengupta S, Banerjee A, Bose SK. $\gamma$-Glutamyl and D-or L-peptide linkages in mycobacillin, a cyclic peptide antibiotic. Biochem J 1971:121:839-46. 
24) Wakayama S, Ishikawa F, Oishi K. Mycocerein, a novel antifungal peptide antibiotic produced by Bacillus cereus. Antimicrob Agents Chemother 1984;26:939-40.

25) Isogai A, Takayama S, Murakoshi S, Suzuki A. Structure of $\beta$-amino acids in antibiotics iturin A. Tetrahedron Lett 1982:23:3065-8.

26) Peypoux F, Besson F, Michel G, Delcambe L. Structure of bacillomycin D, a new antibiotic of the iturin group. Eur J Biochem 1981;118:323-7.

27) Dion HW, Woo PW, Willmer NE, Kern DL, Onaga J, Fusari SA. Butirosin, a new aminoglycosidic antibiotic complex: isolation and characterization. Antimicrob Agents Chemother 1972:2:84-8.

28) Horii S, Nogami I, Mizokami N, Arai Y, Yoneda M. New antibiotic produced by bacteria, 5- $\beta$-D-xylofuranosylneamine. Antimicrob Agents Chemother 1974;5:578-81.

29) Reid WM, Johnson J, Dick J. Anticoccidial activity of lasalocid in control of moderate and severe coccidiosis. Avian Dis 1975:19:12-8.

30) Tewari AK, Maharana BR. Control of poultry coccidiosis: changing trends. J Parasit Dis 2011:35:10-7.

31) Levine R, Horst G, Tonda R, Lumpkins B, Mathis G. Evaluation of the effects of feeding dried algae containing beta-1,3-glucan on broilers challenged with Eimeria. Poult Sci 2018;97:3494-500. 